\title{
Michelson Interferometer design in ECW heated plasmas and initial results
}

\author{
J.W. Oosterbeek, N. Chaudhary, M. Hirsch, M.N.A. Beurskens, U. Höfel, A. Card, M. Marquardt, S. Marsen, M. \\ Stern, R.C. Wolf, and the W7-X team \\ Max-Planck-Institut für Plasmaphysik, Teilinstitut Greifswald, Wendelsteinstraße 1, 17491 Greifswald, Germany
}

\begin{abstract}
The Wendelstein 7-X (W7-X) experiment is equipped with an Electron Cyclotron Resonance Heating installation consisting of 10 gyrotrons capable of delivering up to $7.5 \mathrm{MW}$ of Electron Cyclotron Wave power at the $140 \mathrm{GHz}$ resonance in the plasma. During the plasma discharge the gyrotron power is delivered in a narrow band of several $100 \mathrm{MHz}$ around the gyrotron frequency. At plasma start-up, i.e. initially in the absence of plasma, the non-absorbed gyrotron power in the vessel ('stray radiation') is very high. During the plasma discharge the stray radiation may also rise depending on the plasma optical depth or due to the challenge to deliver the gyrotron power at the exact required polarisation. At W7-X an additional issue is that at high electron density the second harmonic extra-ordinary heating scheme (X2) is no longer possible and one switches to second harmonic ordinary (O2) heating. But the ECH absorption is not complete in the $\mathrm{O} 2$ heating scheme and stray radiation is significantly increased. The stray radiation issue is a problem for sensitive microwave receivers, typically measuring power levels in the sub $\mu \mathrm{W}$ range. For protection, these generally employ notch filters such as fundamental mode interference cavities [1], [2]. This paper focuses on the effect of non-absorbed $140 \mathrm{GHz}$ gyrotron power on the Michelson Interferometer at W7-X.
\end{abstract}

Keywords: ECE, Michelson Interferometer, Gyrotron, Stray radiation, Notch Filter, W7-X

\section{Introduction}

A refurbished Michelson Interferometer (MI) has been taken into operation at the Wendelstein 7-X Stellarator (W7-X). The instrument is commissioned to measure in both $\mathrm{O}$ - and X-mode from $50 \mathrm{GHz} \cdots 300 \mathrm{GHz}$. The frequency span allows to probe multiple ECE harmonics such that it may be used in high density plasmas when the 2-nd harmonic channels of the radiometer go into cutoff and to obtain insight in frequency down shifted ECE. The principle heating scheme at W7-X is by means of 10 gyrotrons operating at $140 \mathrm{GHz}$ with a combined power of 7.5 MW. The ECE power, in contrast, is many orders of magnitude lower. Stray radiation potentially leads to damage or non-linearity of the detector. The subject of this paper is assessment of stray radiation levels and mitigation measures such as a notch filter [4].

\section{Description of the overall MI system}

The ECE signal from the W7-X vessel is collected with a $36 \mathrm{~mm}$ diameter horn antenna by means of a set of quasi-optical in-vessel mirrors. The waveguide following the antenna is tapered to $4 \mathrm{~mm}$ diameter where it is incident upon a vacuum window made with a $100 \mu \mathrm{m}$ thick Mica disk designed for operation in D-band by the X2-mode Radiometer. The cut-off frequency of a section of circular waveguide with diameter $4 \mathrm{~mm}$ is $44 \mathrm{GHz}$ such that it still passes the 1-st ECE harmonic (see Fig 1) but the margin is small and only one mode can pass. At the atmospheric side the waveguide is tapered to $28 \mathrm{~mm}$ diameter circular waveguide and fed to a beam splitter which separates X-mode and O-mode. Both waveguides continue in $28 \mathrm{~mm}$ diameter circular waveguide over 21 $\mathrm{m}$ and 9 mitre bends to the diagnostic hall. By means of waveguide switches the X-mode waveguide normally connects to the radiometer $(120 \mathrm{GHz} \cdots 160 \mathrm{GHz})$, while the O-mode waveguide connects to the MI. The switches allow to swap the waveguides. For the O-mode waveguide the transmission line losses as measured with the (multimode) MI are $11.0+/-0.5 \mathrm{~dB}$. By including invessel losses, the vacuum window and losses in the optics coupling to the MI, the overall loss from plasma to the input of the MI is estimated at $15 \mathrm{~dB}$. The MI itself is of the Martin-Puplett type [5] and is in fact a duplicate of the system in use at JET [6]. It has a $63.5 \mathrm{~mm}$ diameter aperture and waveguide system intersected with cubes 
containing polarising wire grids, such that two optical paths are created: one via a fixed mirror and one via a moveable mirror. The sum of the two signals is incident on a Helium cooled InSb bolometer detector. The excursion of the moveable mirror is $17 \mathrm{~mm}$ and a round trip, taking two interferograms, takes $45 \mathrm{~ms}$. The frequency resolution at $17 \mathrm{~mm}$ mirror excursion is around $5 \mathrm{GHz}$. Using an overall video gain of 50000, a High Pass filter at $1 \mathrm{~Hz}$ and a Low Pass filter at $30 \mathrm{kHz}$, the instrument resolves fluctuations in power in the lower $\mathrm{nW}$ range without averaging. At the other extreme, the manufacture of the detector deems a power level of $1 \mathrm{~mW}$ still acceptable before it gets damaged. This level is adapted here as maximum during error conditions.

\section{Expected power levels}

To assess the effect of ECH power onto the MI, both a measure of the absolute ECH power, and the ratio of $\mathrm{ECH}$ to ECE power are required. These levels will vary with the ECH heating scheme (X2 or O2), the amount of ECH power launched and plasma conditions such as temperature and optical thickness. The focus will be on the $\mathrm{O} 2$ heating scheme as the microwaves are not fully absorbed in such case leading to highest levels of stray radiation (with the exception of plasma start-up and refracted beams).

\subsection{ECE}

Fig. 1 shows a simulated temperature profile for a plasma with a magnetic field on axis $B_{T}=2.5 \mathrm{~T}$, a central electron temperature $T_{e}=5 \mathrm{keV}$ and with a central electron density $n_{e}=5 \cdot 10^{19} \mathrm{~m}^{-3}$, assuming parabolic profiles. Using the Rayleigh-Jeans approximation of the Planck function describing black-body radiation, and taking out the solid angle dependence by ensuring the antenna pattern of the horn antenna is fully filled [7], the total power is $\mathrm{k} \cdot T \cdot B$, with $\mathrm{k}$ the Boltzmann constant, $T$ the temperature and $B$ the bandwidth. The total expected power is the integral over the O-mode spectrum in Fig. 1 But as $\mathrm{O} 2$ is optically thin, only the $\mathrm{O} 1$ contribution is taken and is of the order of $30 \mu \mathrm{W}$. Taking into account the $15 \mathrm{~dB}$ losses, the power at the input of the MI is estimated to be around $1 \mu \mathrm{W}$.

\section{2. $E C H$}

ECH stray radiation levels are highest in the absence of a plasma or in case the plasma is in cut-off for the gyrotron frequency. In case of cut-off a worst case scenario would be direct reflection of a refracted $\mathrm{ECH}$ beam onto

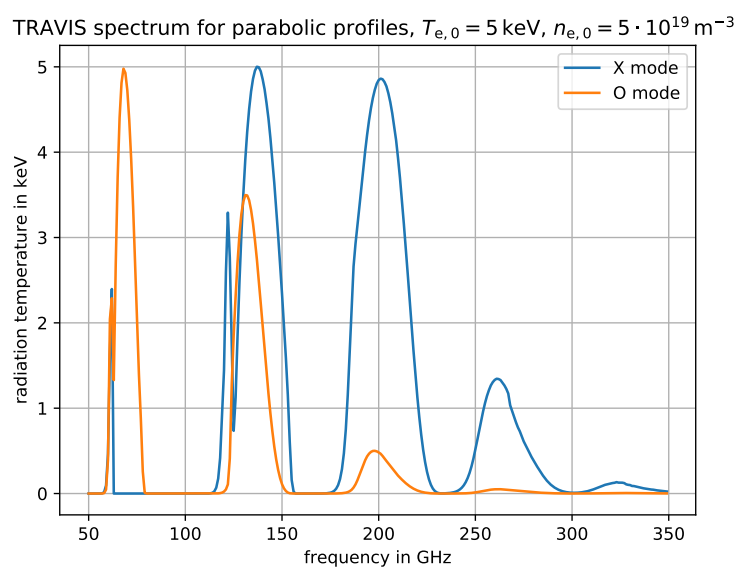

Figure 1: Expected ECE spectra from Travis for X-mode and O-mode.

the ECE antenna, however, this is excluded by the authors given the toroidal distribution of the ECH launchers with respect to the ECE antenna and given the Stellarator vessel geometry. The stray radiation levels $p\left[\mathrm{~kW} / \mathrm{m}^{2}\right]$ in a empty vessel are hard to estimate due to standing waves, however, the vessel can be considered a resonator: by equating the $\mathrm{ECH}$ input power with wall losses $(p \cdot A \cdot \alpha$, with $\mathrm{A}=$ surface, $\alpha=$ absorption coefficient), a power balance results. When a homogeneous distribution is assumed and taking W7-X vessel data, the ECE antenna could be exposed to the order of $p=0.1 \mathrm{MWm}^{-2}$. In $5 \mathrm{MW} \mathrm{O} 2$ heated plasmas - depending on plasma temperature and density - stray radiation levels at the location of the ECE antenna are estimated at $\approx 5 \mathrm{kWm}^{-2}$ using sniffer probes [8].

The total power $P$ for an antenna collecting over all angles would be $P=p \cdot A$, with $A$ the surface of the horn antenna. But the coupled power is reduced by the antenna directivity, which is $\approx 20 \mathrm{~dB}$ for the ECE horn. On entering the horn aperture the number of possibly modes at $140 \mathrm{GHz}$ is about 700 [9]. But the aperture diameter is reduced to $4 \mathrm{~mm}$ over the section of waveguide leading towards the vacuum window, supporting only 9 modes, i.e. a further reduction by $\approx 20 \mathrm{~dB}$. These coupling losses are broad estimates and must be experimentally verified. Finally, the $15 \mathrm{~dB}$ losses from window to instrument are added giving a total reduction of $55 \mathrm{~dB}$.

In an empty vessel, taking a safety marigin of a factor 3, at $5 \mathrm{MW}$ ECH input power and assuming a (fictitious) homogeneous distribution, $p=0.3 \mathrm{MWm}^{-2}$. The expected power amounts in such case to $\approx 1 \mathrm{~mW}$ at the input of the MI. A mechanical shutter was introduced to block the waveguide during RF start-up. In case of plasma operation, using $\mathrm{O} 2$-heating and taking 
$p=5 \mathrm{kWm}^{-2}$, one finds $\approx 15 \mu \mathrm{W}$, i.e. 15 times more then the expected ECE power.

\section{Notch filter}

While the stray radiation levels reported in the previous section will not destroy the detector, a notch filter is required to eliminate the large stray radiation component from the low power ECE signal. To assess the impact of the $140 \mathrm{GHz}$ component on the spectrum a large signal response test was performed.

The MI was illuminated with a hot source (HS) at 600 ${ }^{\circ} \mathrm{C}$ while in a second input port of the MI a RF power signal was injected. The result of this test in shown and discussed in Fig. 2 It is concluded that the notch filter is essential as the overall instrument response is too much affected by excessive ECH power. An interference notch filter based on multiple anti-resonant PTFE plates of 3/4 $\lambda$ was developed and used in the measurement campaign [4]. The notch width as measured in the laboratory is around $10 \mathrm{GHz}$ FWHM. It is noted though that initial coupling optics used in front of the MI were different, which may have resulted in broader notches. Using a RF-source, a rejection at $140 \mathrm{GHz}$ of $34 \mathrm{~dB}$ was measured and using a hot source an insertion loss of $6 \mathrm{~dB}$ was measured. At the time of writing it was not yet possible to characterise the filter over the full band in situ, however, given the design based on $3 / 4 \lambda$ plates, notches should be present at odd multiples of (140/3) GHz. The loss of a significant fraction of the 2-nd harmonic ECE is presently the price paid to probe the multiple harmonics. However, an improved notch filter with $1 / 4 \lambda$ ceramic plates is scheduled, with the aim to reduce the number of notches, the notch width and the insertion loss.

\section{Measurement data}

Fig. 3 shows the MI time response of discharge 20181017.038 with $5 \mathrm{MW}$ heating in $\mathrm{X} 2, B_{T}=$ $2.45 \mathrm{~T}, T_{e}=6 \mathrm{keV}$ and $n_{e}=3 \cdot 10^{19} \mathrm{~m}^{-3}$. In this discharge the notch filter was purposely removed to assess stray radiation levels. The overall video gain was reduced from 50000 to 5000 . Fig 3 a shows the overall discharge and Fig $3 \mathrm{~b}$ a zoom at $t=10 \mathrm{~s}$ when the pulse is terminated by switching the ECH off. $140 \mathrm{GHz}$ induced interference fringes (refer to caption) are present before $t=10 \mathrm{~s}$, while they disappear after $t=10 \mathrm{~s}$. By scaling the amplitude of the $140 \mathrm{GHz}$ fringes to the amplitude of interferograms obtained with the calibrated RF-source, the $\mathrm{ECH}$ power is assessed at $\approx 1 \mu \mathrm{W}$. The ECE power is harder to assess given the large $\mathrm{ECH}$ contribution in the

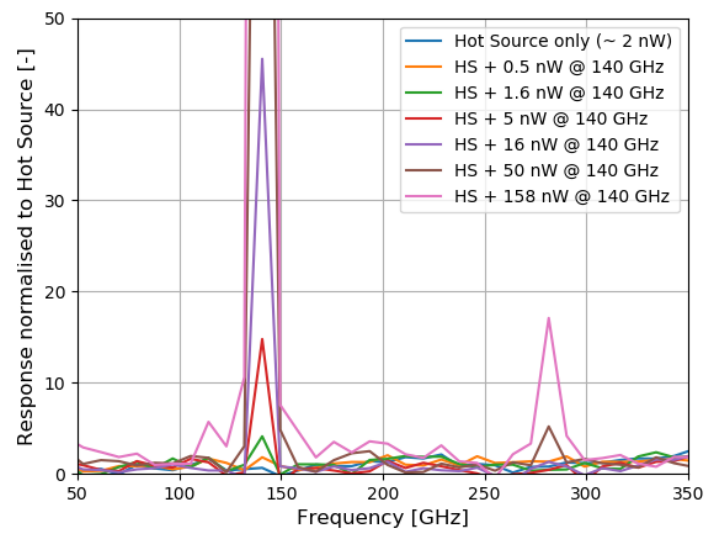

Figure 2: Compression test. The MI is illuminated with a Hot Source (HS) at $600{ }^{\circ} \mathrm{C}$, yielding an expected power of $\approx 2.5 \mathrm{nW}$ taking a bandwidth of $350 \mathrm{GHz}$. RF power is injected at $140 \mathrm{GHz}$ at increasing power levels. Although the RF power is contained in a band of only $100 \mathrm{MHz}$, the effect on the spectrum becomes quite large due to the limited spectral response of the instrument. At $150 \mathrm{nW}$ the $140 \mathrm{GHz}$ spills over to other frequencies. The feature at $280 \mathrm{GHz}$ is a harmonic of the RF source.

interferograms. Considering the interferogram around $\mathrm{t}=10.04 \mathrm{~s}$ and assuming a W7X confinement time of $100 \mathrm{~ms}$, one could arrive at a power of $0.1 \mu \mathrm{W}$ to $0.5 \mu \mathrm{W}$.
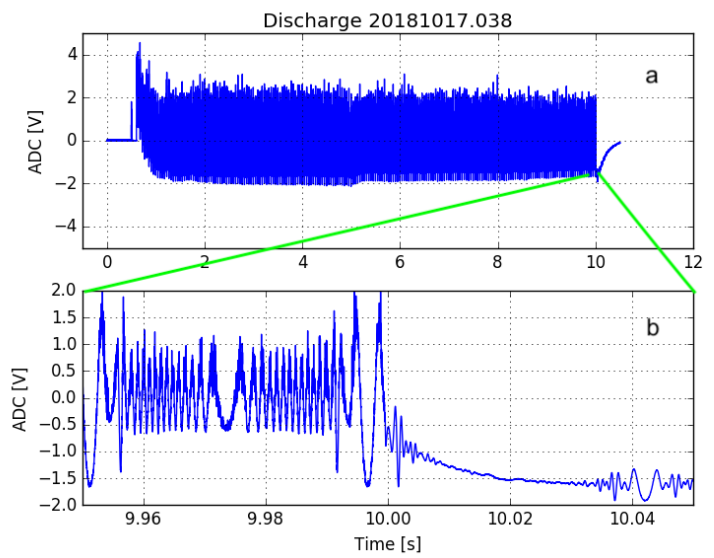

Figure 3: X2 heated pulse 20181017.038 without notch filter. The trace shows $1 \mathrm{MHz}$ time sampled data from the detector, post processed with a reduction factor 10 . In the zoom (Fig $3 \mathrm{p}$ ) the mirror turn around positions are recognised by the slow variations in time. The period up to $10 \mathrm{~s}$ shows a sweep of the mirror back and forth, resulting in an optical path difference of $2 \times 17 \mathrm{~mm} \times 2=68 \mathrm{~mm}$. Approximately 34 fringes are counted resolving a wavelength of $\approx 2.0 \mathrm{~mm}$ or $\approx 140 \mathrm{GHz}$. Note that a concise calculation is performed using mirror metrology data. After $t=10 \mathrm{~s}$, when the ECH is off and the plasma decays, the strong $140 \mathrm{GHz}$ component has disappeared.

To assess O2-heated plasmas, pulse 20181011.034 
was investigated with $B_{T}=2.52 \mathrm{~T}, 5 \mathrm{MW}$ heating in $\mathrm{O} 2$, $T_{e}=2 \mathrm{keV}$ and $n_{e}=(6 \cdots 9) \cdot 10^{19} \mathrm{~m}^{-3}$. The notch filter was in place during the first half of the pulse, and was removed during the second half. In the first half of the pulse, the interferograms are similar to the ECH-free interferogram at $t=10.04 \mathrm{~s}$ in Fig $3 \mathrm{~b}$, while in the second half the interferograms are completely dominated by ECH stray radiation. Following the same methodology as in the $\mathrm{X} 2$-heated pulse, the $140 \mathrm{GHz}$ component is estimated at $16 \mu \mathrm{W}$ and the ECE at $\approx 0.5 \mu \mathrm{W}$.

Fig. 4 shows the spectrum of discharge 20181011.034 with notch filter. The resonse around the 2-nd harmonic (refer to Fig. 1) is reduced, but over a larger section as expected. The same applies to the 1-st harmonic. This may be due to a broader notch width as noted in section 4. To illustrate the response withouth a filter and no ECH, Fig 5 shows a spectrum of a pulse with a Neutral Beam Injection heating phase only $\left(B_{T}=2.47 \mathrm{~T}, 3 \mathrm{MW}\right.$ $\mathrm{NBI}, T_{e}=1 \mathrm{keV}, n_{e}=4 \cdot 10^{19} \mathrm{~m}^{-3}$ ). The differences in the discharges does not allow an one to one comparison but the absence of notches and the reduced losses result in an improved spectrum in Fig. 5, calling for notch filter improvements. Note that as Radiometer Local Oscillator leakage at $244 \mathrm{GHz}$ enters the spectrum, a post plasma spectrum is subtracted to eliminate this feature.

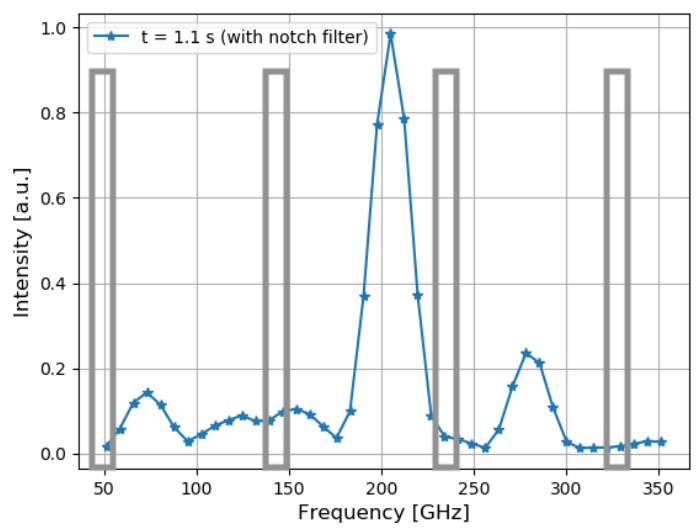

Figure 4: Spectrum of O2 heated pulse 20181011.034 with the notch filter. The notches are shown in grey.

\section{Summary}

First broad band ECE measurements using a Michelson Interferometer have been demonstrated in ECRH O2-heated plasmas enhancing the ECE diagnostic potential at $\mathrm{W} 7-\mathrm{X}$. The potential problem of stray radiation has been investigated and understood. The

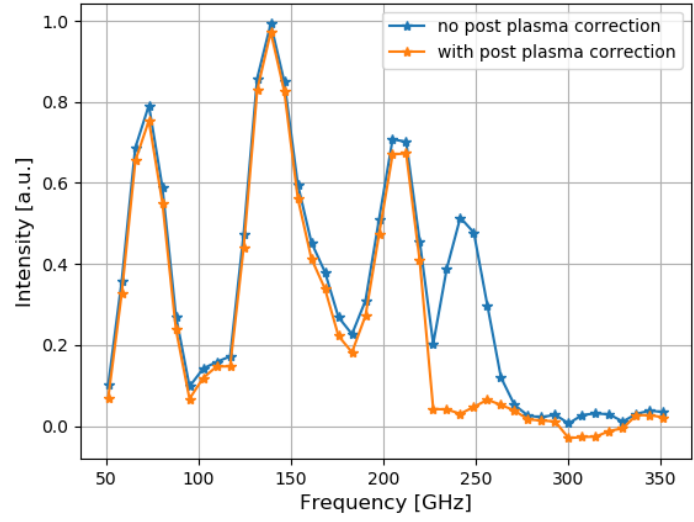

Figure 5: Spectrum of discharge 20181009.018 after $\mathrm{t}=1.5 \mathrm{~s}$ during the NBI-heating only phase and without the notch filter.

stray radiation is suppressed by a broad band proto-type notch filter, which will be further improved.

The authors kindly acknowledge Dr. A. Krämer-Flecken, FZJ Germany, for use of the Hot Source in these measurements. This work has been carried out within the framework of the EUROfusion Consortium and has received funding from the Euratom research and training programme 2014-2018 under grant agreement No 633053. The views and opinions expressed herein do not necessarily reflect those of the European Commission.

\section{References}

[1] M. Moseev et al., Collective Thomson Scattering Diagnostic at Wendelstein 7-X, submitted to Review of Scientific Instruments spring 2018.

[2] V. Furtula et al.,105-GHz Notch Filter Design for Collective Thomson Scattering, Fusion Science and Technology, 59:4, 670677, DOI: 10.13182/FST11-A11732

[3] M. Hirsch et al,. ECE Diagnostic and Measurements during initial Operation of Wendelstein 7-X, 20th Joint Workshop on Electron Cyclotron Emission and Electron Cyclotron Resonance Heating, May 14-17, 2018, Greifswald, Germany

[4] N. Chaudhary et al,. Investigation of Optically Grey Electron Cyclotron Harmonics in Wendelstein 7-X, 20th Joint Workshop on Electron Cyclotron Emission and Electron Cyclotron Resonance Heating, May 14-17, 2018, Greifswald, Germany

[5] D. H. Martin and E. Puplett, Infrared Phys. 10, 105 (1969).

[6] S. Schmuck et al., Electron cyclotron emission spectra in X-and O-mode polarisation at JET, Review of Scientific Instruments 87, 093506 (2016)

[7] Hartfuss, Hans-Jürgen, Fusion Plasma Diagnostics with mmWaves (Wiley-VCH, 2013)

[8] S. Marsen, Private communication.

[9] Nikitin et al., Proceedings of the 2011 IEEE International Symposium on Antennas and Propagation (APSURSI), 3-8 July 2011, Spokane, WA, USA 